\title{
Erratum to JEMS (Journal of ETA Maritime Science), Volume 5, Issue 1 (2017)
}

\section{Erratum (ER)}

DOI ID: $10.5505 /$ jems.2017.25338

The article "A Practical Tool for Evaluating Refrigeration Systems Using R-134a" has an oversight about DOI ID.

The correct DOI ID is given below;

10.5505/jems.2017.19483

The editorial board of JEMS apologizes for any inconvenience that may result from this oversight. 